\title{
Dynamic Output Feedback Passive Control of Uncertain Switched Stochastic Systems with Time-Varying Delay
}

\author{
Huimei Jia, ${ }^{1}$ Hamid Reza Karimi, ${ }^{2}$ and Zhengrong Xiang ${ }^{1}$ \\ ${ }^{1}$ School of Automation, Nanjing University of Science and Technology, Nanjing 210094, China \\ ${ }^{2}$ Department of Engineering, Faculty of Engineering and Science, University of Agder, 4898 Grimstad, Norway \\ Correspondence should be addressed to Zhengrong Xiang; xiangzr@mail.njust.edu.cn
}

Received 21 January 2013; Accepted 14 March 2013

Academic Editor: Xiaojie Su

Copyright (c) 2013 Huimei Jia et al. This is an open access article distributed under the Creative Commons Attribution License, which permits unrestricted use, distribution, and reproduction in any medium, provided the original work is properly cited.

\begin{abstract}
This paper is concerned with the issues of passivity analysis and dynamic output feedback (DOF) passive control for uncertain switched stochastic systems with time-varying delay via multiple storage functions (MSFs) method. Firstly, based on the MSFs method, a sufficient condition for the existence of the passivity of the underlying system is established in terms of linear matrix inequalities (LMIs). Furthermore, the problem of dynamic output feedback passive control is investigated. Based on the obtained passivity condition, a sufficient condition for the existence of the desired switched passive controller is derived. Finally, a numerical example is presented to show the effectiveness of the proposed method.
\end{abstract}

\section{Introduction}

Switched systems consist of a family of continuous-time or discrete-time subsystems operated by a particular type of switching rule. According to this switching rule, one of these subsystems will be activated along the system trajectory at each instant of time. Switched systems have drawn considerable attention during the past few decades because of their applicability and significance in various areas, such as power electronics, embedded systems, chemical processes, and computer-controlled systems [1-4]. Analysis and synthesis of such systems have been studied in a large number of papers, and some research results have been obtained in [5-18]. For example, stability and stabilization problems were investigated in [5-7], the sliding mode control problem was studied in [8-10], the model reduction problem was developed in $[11,12]$, the filtering problem was considered in [13], output feedback control was discussed in [14-17], and so on. In [18], the authors gave a summary of the recent results about the stability and stabilizability of switched systems. On the other hand, due to the presence of random disturbance, stochastic systems also play an important role in engineering applications and have received much attention during the past decades. Many results on stochastic systems and switched stochastic systems have been reported in [19-23]. Besides, time delays frequently occur in practical systems and are often the source of instability, and it is of significance to study time delay systems. Recently, many useful results on such systems have appeared in [24-27].

It is well known that the Lyapunov function method [28] and the average dwell time approach [29] are widely used to study the stability of switched systems. A common Lyapunov function for all subsystems can guarantee the stability under an arbitrary switching signal [30], but sometimes the common Lyapunov function is difficult to find or even might not exist, so Lyapunov-like functions and multiple Lyapunov functions techniques are used widely to ensure the stability under some certain switching signals.

Recently, passivity has been investigated by many researchers. As a special case of dissipativity, it explains the system's internal energy relationship from the point of energy dissipation, and it means that the loss of energy inside the dynamic system is not more than the energy supplied from outside. The concept of passivity was firstly introduced by Willems [31] and was extended by Hill and Moylan [32]. Passivity has a wide range of applications, not only because of its desirable properties, but also because it is a powerful tool 
to analyze the stabilization of systems. Compared with the commonly used stability theory, the use of passivity presents a new tool to analyze the stability, and according to the definition of passivity, storage functions induced by passivity can provide natural candidates for Lyapunov functions to analyze the Lyapunov stability and stabilization problem of systems. The notion of passivity for a class of switched control systems was developed in [33], and the relationship between Lyapunov stability and passivity was analyzed. Because the common storage function is hard to search, to overcome this restriction, a notion of multiple storage functions (MSFs) was proposed in [34]. MSFs mean that each subsystem has its own storage function, and each storage function is nonincreasing for zero input on the "switched on" time to guarantee the stability of the system. Many scholars have done a lot of research on passivity of switched systems and have achieved fruitful research results [33-38]. A framework of dissipativity theory for switched systems using MSFs and multiple supply rates was set up in [34], a concept of passivity for switched systems using MSFs was presented in [35], and the issues of passivity, feedback equivalence, and stability for switched nonlinear systems via MSFs approach were investigated in $[36,37]$. In [38], the dissipativity-based sliding mode control of switched stochastic systems was studied, and the passivity condition was established by applying the average dwell time approach. It is worth pointing out that the result presented in [38] is mainly on switched stochastic delay-free systems. However, to the best of our knowledge, the passivity analysis and control synthesis for switched stochastic systems with time-varying delay have not been fully investigated to date. This motivates the present study.

In this paper, we focus on the passive analysis and DOF passive control for a class of uncertain switched stochastic systems with time-varying delay. The main contributions of this paper can be summarized as follows: (i) by utilizing the MSFs method, a passivity condition is derived in terms of LMIs; and (ii) based on the result obtained, a DOF passive controller is constructed for the system.

This paper is organized as follows. In Section 2, problem formulation and some necessary lemmas are given. In Section 3, the main results are presented. Section 4 gives a numerical example to illustrate the effectiveness of the proposed approach. Finally, conclusions are provided in Section 5 .

Notations. The notations throughout this paper are quite standard. $R^{n}$ and $R^{n \times m}$ denote the $n$-dimensional Euclidean space and the set of all $n \times m$ real matrices, respectively. $A^{T}$ and $A^{-1}$ denote the transpose and the inverse of any square matrix $A$. The notation $A>0$ means that matrix $A$ is positive definite. $I$ is the identity matrix with an appropriate dimension. The symmetric term in a matrix is denoted by “*." $E\{\cdot\}$ is the expectation operator. $\operatorname{diag}(\cdot)$ denotes a block diagonal matrix. $C_{n, \tau}$ denotes the set of all $R^{n}$ valued continuous functions defined on the interval $[-\tau, 0]$. $L_{2}[0, \infty)$ is the space of square integrable functions on $[0, \infty)$.

\section{Problem Formulation and Preliminaries}

Consider the following uncertain switched stochastic systems with time-varying delay:

$$
\begin{aligned}
d x(t)= & {\left[\bar{A}_{\sigma(t)} x(t)+\bar{A}_{d \sigma(t)} x(t-d(t))+B_{\sigma(t)} u(t)\right.} \\
& \left.+B_{v \sigma(t)} v(t)\right] d t+\bar{S}_{\sigma(t)} x(t) d w(t), \\
z(t)= & C_{1 \sigma(t)} x(t)+D_{1 \sigma(t)} u(t)+D_{1 v \sigma(t)} v(t), \\
d y(t)= & {\left[\bar{C}_{2 \sigma(t)} x(t)+\bar{C}_{2 d \sigma(t)} x(t-d(t))+D_{2 v \sigma(t)} v(t)\right] d t } \\
& +\bar{G}_{\sigma(t)} x(t) d w(t), \\
x(t)= & \phi(t), \quad t \in[-\tau, 0],
\end{aligned}
$$

where $x(t) \in R^{n}$ is the state vector, $u(t) \in R^{m}$ is the control input, $z(t) \in R^{q}$ is the output vector, $v(t) \in R^{p}$ is the disturbance input which belongs to $L_{2}[0, \infty), y(t) \in R^{r}$ is the measured output, and $\phi(t) \in C_{n, \tau}$ represents the initial state function.

$\sigma(t) \in[0, \infty) \rightarrow \underline{N}=\{1,2, \ldots, N\}$ is a piecewise right continuous constant function. The switching sequence can be described as $\Sigma:\left\{\left(t_{0}, \sigma\left(t_{0}\right)\right),\left(t_{1}, \sigma\left(t_{1}\right)\right), \ldots,\left(t_{k}, \sigma\left(t_{k}\right)\right), \ldots\right\}$, where $t_{0}=0$ is the initial time, and $t_{k}$ denotes the $k$ th switching instant. Moreover, $\sigma(t)=i$ means that the $i$ th subsystem is activated. $w(t)$ is a zero-mean Wiener process on a probability space $(\Omega, F, \mathrm{P})$ satisfying

$$
E\{d w(t)\}=0, \quad E\left\{d w^{2}(t)\right\}=d t,
$$

where $\Omega$ is the sample space, $F$ is $\sigma$-algebras of subsets of the sample space, $P$ is the probability measure on $F . d(t)$ is the time-varying delay satisfying

$$
0 \leq d(t) \leq \tau, \quad 0 \leq \dot{d}(t) \leq \mu<1,
$$

where $\tau$ and $\mu$ are known constants. Consider

$$
\begin{aligned}
& \bar{A}_{i}=A_{i}+\Delta A_{i}(t), \quad \bar{A}_{d i}=A_{d i}+\Delta A_{d i}(t), \\
& \bar{S}_{i}=S_{i}+\Delta S_{d i}(t), \quad \bar{C}_{2 i}=C_{2 i}+\Delta C_{2 i}(t), \\
& \bar{C}_{2 d i}=C_{2 d i}+\Delta C_{2 d i}(t), \quad \bar{G}_{i}=G_{i}+\Delta G_{i}(t),
\end{aligned}
$$

where $A_{i}, A_{d i}, B_{i}, B_{v i}, S_{i}, C_{1 i}, D_{1 i}, D_{1 v i}, C_{2 i}, C_{2 d i}, D_{2 v i}$, and $G_{i}$, $i \in N$, are constant matrices with appropriate dimensions, and $\bar{\Delta} A_{i}(t), \Delta A_{d i}(t), \Delta S_{i}(t), \Delta C_{2 i}(t), \Delta C_{2 d i}(t)$, and $\Delta G_{i}(t)$, $i \in \underline{N}$, are unknown matrices representing time-varying parameter uncertainties and assumed to be of the form

$$
\begin{aligned}
& {\left[\begin{array}{ccc}
\Delta A_{i}(t) & \Delta A_{d i}(t) & \Delta S_{i}(t) \\
\Delta C_{i}(t) & \Delta C_{d i}(t) & \Delta G_{i}(t)
\end{array}\right]} \\
& =\left[\begin{array}{l}
M_{1 i} \\
M_{2 i}
\end{array}\right] F_{i}(t)\left[\begin{array}{lll}
N_{1 i} & N_{2 i} & N_{3 i}
\end{array}\right], \quad i \in \underline{N},
\end{aligned}
$$

where $M_{1 i}, M_{2 i}, N_{1 i}, N_{2 i}$, and $N_{3 i}$ are known real constant matrices, and $F_{i}(t)$ is an unknown time-varying matrix function satisfying

$$
F_{i}^{T}(t) F_{i}(t) \leq I .
$$


Definition 1 (see [36]). For system (1) with $u(t)=0$, it is said to be stochastically passive under the switching signal $\sigma(t)$ if there exists a nonnegative function $S_{\sigma(t)}(x)$ with $S_{\sigma(t)}(0)=0$, such that the following inequality holds:

$$
\begin{array}{r}
E\left\{S_{\sigma(t)}(x(t))-S_{\sigma(s)}(x(s))\right\} \leq \int_{s}^{t} E\left\{2 v^{T}(\theta) z(\theta)\right\} d \theta, \\
s \leq t<\infty .
\end{array}
$$

Remark 2. In Definition 1 , the nonnegative function $S_{\sigma(t)}(x)$ is called storage function. Passivity is explained by the system internal energy relationship from the point of energy dissipation; so the stored energy function of the system is called storage function. Usually, a common storage function for all subsystems may not exist or it may be rather difficult to find, but each subsystem may have its own storage function; thus, we consider the passivity under the switching signal via MSFs method in this paper.

Definition 3 (see [36]). Let $S_{i}$ be a positive semidefinite function and assume that the $i$ th subsystem is active during the interval $\left[t_{k}, t_{k+1}\right) . S_{i}$ is called to be the storage-like function of the $i$ th subsystem if the following passivity inequality holds:

$$
\begin{array}{r}
E\left\{S_{i}(x(t))-S_{i}(x(s))\right\} \leq \int_{s}^{t} E\left\{2 v^{T}(\theta) z(\theta)\right\} d \theta \\
t_{k} \leq s \leq t<t_{k+1} .
\end{array}
$$

Remark 4. In Definition 3, inequality (8) indicates that the passivity property of the $i$ th subsystem holds when it is active.

Remark 5. Actually, the stability-theory-based Lyapunov functions can be explained from the vision of passivity. It is often needed to construct Lyapunov functions when we consider the stability of the system; this process can be transformed to the process of constructing the storage functions to make the system passive.

Lemma 6 (see [24]). For any constant matrix $R>0$, scalar $d>0$, and vector function $x(t)$, such that the following Jensen's integral inequality is well defined:

$$
-d \int_{t-d}^{t} x(s) R x(s) d s \leq-\left[\int_{t-d}^{t} x(s) d s\right]^{T} R\left[\int_{t-d}^{t} x(s) d s\right] .
$$

Lemma 7 (see [13]). Let $U, V, W$, and $X$ be real matrices of appropriate dimensions with $X$ satisfying $X=X^{T}$, then for all $V^{T} V \leq I, X+U V W+W^{T} V^{T} U^{T}<0$,

(a) if and only if there exists a scalar $\varepsilon>0$ such that

$$
X+\varepsilon U U^{T}+\varepsilon^{-1} W^{T} W<0 ;
$$

(b) for any positive definite matrix $R$ :

$$
U W+W^{T} U^{T} \leq U R^{-1} U^{T}+V^{T} R V .
$$

Lemma 8 (see [39]). $A, M, N$, and $P$ are real matrices of appropriate dimensions. $F(t)$ is time-varying matrix with $F^{T}(t) F(t) \leq I$. If there exists a scalar $\varepsilon>0$ such that $P-$ $\varepsilon M M^{T}>0$, then we have

$$
\begin{aligned}
(A+M F N)^{T} P(A+M F N) \leq & A^{T}\left(P^{-1}-\varepsilon M M^{T}\right)^{-1} A \\
& +\varepsilon^{-1} N^{T} N .
\end{aligned}
$$

\section{Main Results}

In this section, we first investigate the passivity analysis of system (1); then based on the passive condition obtained, an output feedback controller is designed to guarantee the passivity of the resulting closed-loop system.

\subsection{Passivity Analysis}

Theorem 9. Consider system (1) with $u(t)=0$, the system is stochastically passive for the switching signal $\sigma(t)=\min \{i \mid i=$ $\left.\arg \min _{i \in N} V_{i}(x(t))\right\}$ if there exist symmetric positive matrices $P_{i}, Q_{i}, Z_{i}, R_{i}$ and matrices $L_{i}, i \in \underline{N}$, with appropriate dimensions such that

$$
\left[\begin{array}{ccccccc}
\Xi_{11} & P_{i} \bar{A}_{d i} & 0 & P_{i} B_{v i}-C_{1 i}^{T} & \bar{S}_{i}^{T} P_{i} & \bar{A}_{i}^{T} P_{i} & 0 \\
* & -(1-\mu) Q_{i} & 0 & 0 & 0 & \bar{A}_{d i}^{T} P_{i} & 0 \\
* & * & \Xi_{33} & 0 & 0 & 0 & L_{i} \\
* & * & * & -D_{1 v i}^{T}-D_{1 v i} & 0 & B_{v i}^{T} P_{i} & 0 \\
* & * & * & * & -P_{i} & 0 & 0 \\
* & * & * & * & * & -P_{i} & 0 \\
* & * & * & * & * & * & -P_{i}
\end{array}\right]
$$

where $\Xi_{11}=Q_{i}+\tau Z_{i}+\bar{A}_{i}^{T} P_{i}+P_{i} \bar{A}_{i}, \Xi_{33}=\tau R_{i}-L_{i}^{T}-L_{i}$.

Proof. Let $f(t):=\bar{A}_{\sigma(t)} x(t)+\bar{A}_{d \sigma(t)} x(t-d(t))+B_{v \sigma(t)} v(t)$, and $\eta(t) d t=d x(t)$, when the $i$ th subsystem is activated, we have

$$
2 \eta^{T}(t) L_{i}\left[(f(t)-\eta(t)) d t+\bar{S}_{i} x(t) d w(t)\right]=0
$$

Choose the following Lyapunov functional candidate

$$
\begin{aligned}
V_{i}(x(t), t)= & V_{1 i}(x(t), t)+V_{2 i}(x(t), t)+V_{3 i}(x(t), t) \\
& +V_{4 i}(x(t), t)
\end{aligned}
$$


where

$$
\begin{aligned}
& V_{1 i}(x(t), t)=x^{T}(t) P_{i} x(t), \\
& V_{2 i}(x(t), t)=\int_{t-d(t)}^{t} x^{T}(s) Q_{i} x(s) d s, \\
& V_{3 i}(x(t), t)=\int_{-\tau}^{0} \int_{t+\theta}^{t} x^{T}(s) Z_{i} x(s) d s, \\
& V_{4 i}(x(t), t)=\int_{-\tau}^{0} \int_{t+\theta}^{t} \eta^{T}(s) R_{i} \eta(s) d s .
\end{aligned}
$$

According to the Itô formula, along the trajectory of the system, we have

$$
d V_{i}(x(t), t)=L V_{i}(x(t), t) d t+2 x^{T}(t) P_{i} \bar{S}_{i} x(t) d w(t) .
$$

Substituting (14) into (17), we get

$$
\begin{aligned}
d V_{i}(x(t), t)= & L \widetilde{V}_{i}(x(t), t) d t \\
& +2\left[x^{T}(t) P_{i} \bar{S}_{i} x(t)+\eta^{T}(t) L_{i} \bar{S}_{i} x(t)\right] d w(t),
\end{aligned}
$$

where $L \widetilde{V}_{i}(x(t), t)=L V_{i}(x(t), t)+2 \eta^{T}(t) L_{i}[(f(t)-\eta(t))]$.

Taking the expectation, we have

$$
E\left\{\frac{d V_{i}(x(t), t)}{d t}\right\}=E\left\{L \widetilde{V}_{i}(x(t), t)\right\} .
$$

For convenience, we write $L \widetilde{V}_{i}=L \widetilde{V}_{i}(x(t), t)$; then it can be obtained from (17) that

$$
\begin{aligned}
L \widetilde{V}_{i} \leq & 2 x^{T}(t) P_{i} f(t)+x^{T}(t) \bar{S}_{i}^{T} P_{i} \bar{S}_{i} x(t)+x^{T}(t) Q_{i} x(t) \\
& -(1-\mu) x^{T}(t-d(t)) Q_{i} x(t-d(t))+\tau x^{T}(t) Z_{i} x(t) \\
& +\tau \eta^{T}(t) R_{i} \eta(t) \\
& -\int_{t-d(t)}^{t} x^{T}(s) Z_{i} x(s) d s-\int_{t-d(t)}^{t} \eta^{T}(s) R_{i} \eta(s) d s \\
& +2 \eta^{T}(t) L_{i}[(f(t)-\eta(t))] .
\end{aligned}
$$

It follows that

$$
\begin{aligned}
L \widetilde{V}_{i}(x(t))- & 2 v^{T}(t) z(t) \\
\leq & \xi^{T}(t)\left(\Xi+\Xi_{1}+\Xi_{1}^{T}+\Xi_{2}\right) \xi(t) \\
& \quad-\int_{t-d(t)}^{t}\left[\begin{array}{ll}
x^{T}(s) & \eta^{T}(s)
\end{array}\right]\left[\begin{array}{cc}
Z_{i} & 0 \\
0 & R_{i}
\end{array}\right]\left[\begin{array}{l}
x(s) \\
\eta(s)
\end{array}\right] d s,
\end{aligned}
$$

where

$$
\begin{aligned}
& \xi^{T}(t)=\left[\begin{array}{llll}
x^{T}(t) & x^{T}(t-d(t)) & \eta^{T}(t) & v^{T}(t)
\end{array}\right], \\
& \Xi_{1}=\widetilde{L}_{i} \widetilde{A}, \quad \widetilde{L}_{i}^{T}=\left[\begin{array}{llll}
0 & 0 & L_{i}^{T} & 0
\end{array}\right], \\
& \widetilde{A}=\left[\begin{array}{llll}
\bar{A}_{i} & \bar{A}_{d i} & 0 & B_{v i}
\end{array}\right],
\end{aligned}
$$

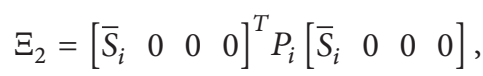

$$
\begin{aligned}
& \Xi=\left[\begin{array}{cccc}
\Xi_{11} & P_{i} \bar{A}_{d i} & 0 & P_{i} B_{v i}-C_{1 i}^{T} \\
* & -(1-\mu) Q_{i} & 0 & 0 \\
* & * & \Xi_{33} & 0 \\
* & * & * & -D_{1 v i}^{T}-D_{1 v i}
\end{array}\right] .
\end{aligned}
$$

By Lemma 7 and Schur complement lemma, we can obtain from (13) that

$$
\Xi+\Xi_{1}+\Xi_{1}^{T}+\Xi_{2}<0
$$

then the following inequality can be obtained:

$$
E\left\{\frac{d V_{i}(x(t), t)}{d t}\right\}=E\left\{L V_{i}(x(t), t)\right\}<2 E\left\{v^{T}(t) z(t)\right\} .
$$

For $t_{k} \leq s<t<t_{k+1}$, the following inequality holds:

$$
E\left\{V_{\sigma\left(t_{k}\right)}(x(t), t)-V_{\sigma\left(t_{k}\right)}(x(s), s)\right\}<\int_{s}^{t} E\left\{2 v^{T}(s) z(s)\right\} d s .
$$

When the switching signal is chosen as $\sigma(t)=\min \{i \mid i=$ $\left.\arg \min _{i \in \underline{N}} V_{i}(x(t))\right\}$, for $t_{k} \leq t<t_{k+1}$, one obtains

$$
\begin{aligned}
& E\left\{V_{\sigma\left(t_{k}\right)}(x(t), t)-V_{\sigma\left(t_{0}\right)}(x(0), 0)\right\} \\
&=E\left\{\int_{0}^{t_{1}} L V_{\sigma\left(t_{0}\right)}(x(s), s) d s+\int_{t_{1}}^{t_{2}} L V_{\sigma\left(t_{1}\right)}(x(s), s) d s\right. \\
&\left.+\cdots \int_{t_{k}}^{t} V_{\sigma\left(t_{k}\right)}(x(t), t) d s\right\} \\
&+ E\left\{\sum_{g=1}^{k}\left(V_{\sigma\left(t_{g}\right)}\left(x\left(t_{g}\right), t_{g}\right)-V_{\sigma\left(t_{g-1}\right)}\left(x\left(t_{g}\right), t_{g}\right)\right)\right\} \\
&<\int_{0}^{t} E\left\{2 v^{T}(s) z(s)\right\} d t .
\end{aligned}
$$

This completes the proof.

Remark 10. The passive condition presented in Theorem 9 is delay dependent, and it is obtained by constructing an appropriate multiple Lyapunov functions. Some other methods dealing with time delay systems, such as delay divisioning method and input-output method, can also be utilized to investigate the systems under consideration, and this will be our further work.

When the matrix parameters in system (1) are determined, according to Theorem 9 , we have the following result. 
Corollary 11. Consider the nominal system in (1), it is stochastically passive under the switching signal $\sigma(t)=\min \{i \mid i=$ $\left.\arg \min _{i \in N} V_{i}(x(t))\right\}$ if there exist symmetric positive matrices $P_{i}, Q_{i}, R_{i}$ and matrices $L_{i}, i \in \underline{N}$, with appropriate dimensions such that

$$
\left[\begin{array}{ccccccc}
\Gamma_{11} & P_{i} A_{d i} & 0 & P_{i} B_{v i}-C_{1 i}^{T} & S_{i}^{T} P_{i} & A_{i}^{T} P_{i} & 0 \\
* & -(1-\mu) Q_{i} & 0 & 0 & 0 & A_{d i}^{T} P_{i} & 0 \\
* & * & \Gamma_{33} & 0 & 0 & 0 & L_{i} \\
* & * & * & -D_{1 v i}^{T}-D_{1 v i} & 0 & B_{v i}^{T} P_{i} & 0 \\
* & * & * & * & -P_{i} & 0 & 0 \\
* & * & * & * & * & -P_{i} & 0 \\
* & * & * & * & * & * & -P_{i}
\end{array}\right]<0,
$$

where $\Gamma_{11}=Q_{i}+\tau Z_{i}+A_{i}^{T} P_{i}+P_{i} A_{i}, \Gamma_{33}=\tau R_{i}-L_{i}^{T}-L_{i}$.

3.2. DOF Passivity Analysis. In this subsection, we focus on designing a DOF passive controller for system (1) such that the corresponding closed-loop system is stochastically passive.

Consider a full-order DOF passive controller with the following form:

$$
\Sigma_{k}:\left\{\begin{array}{l}
d \widehat{x}(t)=A_{k \sigma(t)} \widehat{x}(t) d t+B_{k \sigma(t)} d y(t) \\
u(t)=C_{k \sigma(t)} \widehat{x}(t)
\end{array}\right.
$$

where $\widehat{x} \in R^{n}$ is the controller state; $A_{k i}, B_{k i}$, and $C_{k i}, i \in \underline{N}$, are matrices to be determined.

Applying this controller to system (1), we obtain the following closed-loop system:

$\Sigma_{c}:\left\{\begin{aligned} d \xi(t)= & {\left[\bar{A}_{c i} \xi(t)+\bar{A}_{c d i} H \xi(t-d(t))+B_{c v i} v(t)\right] d t } \\ & +\bar{S}_{c i} \xi(t) d w(t) \\ z(t)= & C_{c i} \xi(t)+D_{c v i} v(t),\end{aligned}\right.$

where

$$
\begin{aligned}
& \xi^{T}(t)=\left[\begin{array}{ll}
x^{T}(t) & \left.\hat{x}^{T}(t)\right]
\end{array}\right. \\
& \bar{A}_{c i}=A_{c i}+\Delta A_{c i}(t), \quad \bar{A}_{c d i}=A_{c d i}+\Delta A_{c d i}(t), \\
& \bar{S}_{c i}=S_{c i}+\Delta S_{c i}(t), \quad H=\left[\begin{array}{ll}
I & 0
\end{array}\right], \\
& \bar{A}_{c i}=\left[\begin{array}{cc}
\bar{A}_{i} & B_{i} C_{k i} \\
B_{k i} \bar{C}_{2 i} & A_{k i}
\end{array}\right], \quad \bar{A}_{c d i}=\left[\begin{array}{c}
\bar{A}_{d i} \\
B_{k i} \bar{C}_{2 d i}
\end{array}\right] \text {, } \\
& B_{c v i}=\left[\begin{array}{c}
B_{v i} \\
B_{k i} D_{2 v i}
\end{array}\right], \quad \bar{S}_{c i}=\left[\begin{array}{c}
\bar{S}_{i} \\
B_{k i} \bar{G}_{i}
\end{array}\right] H,
\end{aligned}
$$

$$
\begin{gathered}
C_{c i}=\left[\begin{array}{cc}
C_{1 i} & D_{1 i} C_{k i}
\end{array}\right], \quad D_{c v i}=D_{1 v i} \\
\widetilde{M}_{1 i}=\left[\begin{array}{c}
M_{1 i} \\
B_{k i} M_{2 i}
\end{array}\right], \quad \widetilde{N}_{1 i}=N_{1 i} \\
\widetilde{N}_{2 i}=N_{2 i}, \quad \widetilde{N}_{3 i}=N_{3 i} .
\end{gathered}
$$

Then, the output feedback passive controller design problem can be converted into the problem of passivity analysis of the closed-loop system (29).

Theorem 12. Consider system (29), it is stochastically passive for the switching signal $\sigma(t)=\min \left\{i \mid i=\arg \min _{i \in N} V_{i}(x(t))\right\}$ if there exist symmetric positive matrices $P_{i}, Q_{i}, R_{i}$ and matrices $L_{c i}, i \in N$, with appropriate dimensions such that the following matrix inequality holds:

$$
\left[\begin{array}{ccccccc}
\Theta_{11} & P_{i} \bar{A}_{c d i} & 0 & P_{i} B_{c v i}-C_{c i}^{T} & \bar{S}_{c i}^{T} P_{i} & \bar{A}_{c i}^{T} P_{i} & 0 \\
* & -(1-\mu) Q_{i} & 0 & 0 & 0 & \bar{A}_{c d i}^{T} P_{i} & 0 \\
* & * & \Theta_{33} & 0 & 0 & 0 & L_{c i} \\
* & * & * & -D_{c v i}^{T}-D_{c v i} & 0 & B_{c v i}^{T} P_{i} & 0 \\
* & * & * & * & -P_{i} & 0 & 0 \\
* & * & * & * & * & -P_{i} & 0 \\
* & * & * & * & * & * & -P_{i}
\end{array}\right]
$$

where $\Theta_{11}=H^{T} Q_{i} H+\tau H^{T} Z_{i} H+\bar{A}_{c i}^{T} P_{i}+P_{i} \bar{A}_{c i}, \Theta_{33}=$ $\tau^{2} H^{T} R_{i} H-L_{c i}^{T}-L_{c i}$.

Proof. Let $g(t):=\bar{A}_{c i} \xi(t)+\bar{A}_{c d i} \xi(t-d(t))+B_{c v i} v(t)$ and $\varsigma(t) d t=d \xi(t)$, then we have

$$
2 \varsigma^{T}(t) L_{c i}\left[(g(t)-\varsigma(t)) d t+\bar{S}_{c i} \xi(t) d w(t)\right]=0 .
$$

Choose the following Lyapunov functional candidate:

$$
\begin{aligned}
& V_{i}(\xi(t), t) \\
& =\xi^{T}(t) P_{i} \xi(t)+\int_{t-d(t)}^{t} \xi^{T}(s) H^{T} Q_{i} H \xi(s) d s \\
& \quad+\int_{-\tau}^{0} \int_{t+\theta}^{t} \xi^{T}(s) H^{T} Z_{i} H \xi(s) d s \\
& \quad+\int_{-\tau}^{0} \int_{t+\theta}^{t} \varsigma^{T}(s) H^{T} R_{i} H \varsigma(s) d s .
\end{aligned}
$$

From the relation between $\xi(t)$ and $x(t)$, we obtain

$$
\begin{gathered}
H \xi(t)=x(t) \\
H \xi(t-d(t))=x(t-d(t)) .
\end{gathered}
$$


Denote $\zeta^{T}(t)=\left[\xi^{T}(t) x^{T}(t-d(t)) \varsigma^{T}(t) v^{T}(t)\right]$, then following the proof line of Theorem 9 , it can be obtained form (31) that

$$
L V_{i}(\xi(t), t)-2 v^{T}(t) z(t)<0 .
$$

It follows that

$$
E\left\{\frac{d V_{i}(\xi(t), t)}{d t}\right\}=E\left\{L V_{i}(\xi(t), t)\right\}<2 E\left\{v^{T}(t) z(t)\right\} .
$$

For $t_{k} \leq s<t<t_{k+1}$, one obtains

$$
E\left\{V_{\sigma(k)}(\xi(t), t)-V_{\sigma(k)}(\xi(s), s)\right\}<\int_{s}^{t} E\left\{2 v^{T}(s) z(s)\right\} d s .
$$

Under the switching signal $\sigma(t)=\min \left\{i \mid i=\arg \min _{i \in \underline{N}} V_{i}\right.$ $(x(t))\}$, for $t_{k} \leq t<t_{k+1}$, we obtain

$$
\begin{aligned}
& E\left\{V_{\sigma\left(t_{k}\right)}(\xi(t), t)-V_{\sigma\left(t_{0}\right)}(\xi(0), 0)\right\} \\
&=E\left\{\int_{0}^{t_{1}} L V_{\sigma\left(t_{0}\right)}(\xi(s), s) d s+\int_{t_{1}}^{t_{2}} L V_{\sigma\left(t_{1}\right)}(\xi(s), s) d s\right. \\
&\left.+\cdots \int_{t_{k}}^{t} V_{\sigma\left(t_{k}\right)}(\xi(t), t) d s\right\} \\
&+ E\left\{\sum_{g=1}^{k}\left(V_{\sigma\left(t_{g}\right)}\left(\xi\left(t_{g}\right), t_{g}\right)-V_{\sigma\left(t_{g-1}\right)}\left(\xi\left(t_{g}\right), t_{g}\right)\right)\right\} \\
&<\int_{0}^{t} E\left\{2 v^{T}(s) z(s)\right\} d t .
\end{aligned}
$$

This completes the proof.

3.3. DOF Passivity Controller Design. In Theorem 12, we have obtained a sufficient condition under which the closedloop system $\sum_{c}$ is stochastically passive, but the designed parameters have not been given. The following theorem presents a controller design method and gives all parameters of the controller.

Theorem 13. Consider system (1), there exists a controller (28) such that the closed-loop system $\sum_{c}$ is stochastically passive for the switching signal $\sigma(t)=\min \left\{i \mid i=\arg \min _{i \in N} V_{i}(x(t))\right\}$ if there exist scalars $\varepsilon>0, \beta>0$, and positive definite symmetric matrices $\Omega_{i}, Y_{i}, Q_{i}, R_{i}, Z_{i}$ and matrices $X_{i}, Y_{i}, T_{i}, i \in \underline{N}$, with appropriate dimensions such that, for all $i \in \underline{N}$,

$$
\left[\begin{array}{cccccccc}
\Psi_{11} & \Psi_{12} & 0 & \Psi_{14} & \Psi_{15} & \Psi_{16} & 0 & U_{1} \\
* & \Psi_{22} & 0 & 0 & 0 & \Psi_{26} & 0 & U_{2} \\
* & * & 2 \beta \psi & 0 & 0 & 0 & -\beta \psi & U_{3} \\
* & * & * & \Psi_{44} & 0 & \Psi_{46} & 0 & 0 \\
* & * & * & * & \psi & 0 & 0 & U_{1} \\
* & * & * & * & * & \psi & 0 & 0 \\
* & * & * & * & * & * & \psi & U_{4} \\
* & * & * & * & * & * & * & V
\end{array}\right]<0,
$$

where

$$
\begin{aligned}
& \Psi_{11}=\left[\begin{array}{cc}
A_{i} \Omega_{i}+B_{i} Y_{i}+\Omega_{i} A_{i}^{T}+Y_{i}^{T} B_{i}^{T} & A_{i}+T_{i}^{T} \\
* & \Upsilon_{i} A_{i}+X_{i} C_{2 i}+A_{i}^{T} \Upsilon_{i}+C_{2 i}^{T} X_{i}^{T}
\end{array}\right], \\
& \Psi_{12}=\left[\begin{array}{c}
A_{d i} \\
\Upsilon_{i} A_{d i}+X_{i} C_{2 d i}
\end{array}\right], \\
& \Psi_{14}=\left[\begin{array}{l}
B_{v i}-\Omega_{i} C_{1 i}^{T}-Y_{i}^{T} D_{1 i}^{T} \\
\Upsilon_{i} B_{v i}+X_{i} D_{2 v i}-C_{1 i}^{T}
\end{array}\right],
\end{aligned}
$$$$
\Psi_{15}=\left[\begin{array}{cc}
\Omega_{i} S_{i}^{T} & 0 \\
S_{i}^{T} & S_{i}^{T} \Upsilon_{i}+G_{i}^{T} X_{i}
\end{array}\right]
$$$$
\Psi_{16}=\left[\begin{array}{cc}
\Omega_{i} A_{i}^{T}+Y_{i}^{T} B_{i}^{T} & T_{i}^{T} \\
A_{i}^{T} & A_{i}^{T} \Upsilon_{i}+C_{2 i}^{T} X_{i}^{T}
\end{array}\right],
$$$$
\Psi_{22}=-(1-\mu) Q_{i}, \quad \Psi_{44}=-D_{1 v i}^{T}-D_{1 v i} \text {, }
$$$$
\Psi_{26}=\left[\begin{array}{ll}
A_{d i}^{T} & \left.A_{d i}^{T} \Upsilon_{i}+C_{2 d i}^{T} X_{i}^{T}\right]
\end{array}\right.
$$$$
\Psi_{46}=\left[\begin{array}{ll}
B_{v i}^{T} & B_{v i}^{T} \Upsilon_{i}+D_{2 v i}^{T} X_{i}^{T}
\end{array}\right], \quad \psi=\left[\begin{array}{cc}
-\Omega_{i} & -I \\
-I & -\Upsilon_{i}
\end{array}\right],
$$$$
U_{1}=\left[\begin{array}{ccccccccc}
M_{1 i} & 0 & 0 & \Omega_{i} & \Omega_{i} N_{1 i}^{T} & \Omega_{i} N_{3 i}^{T} & \Omega_{i} & \Omega_{i} & 0 \\
\Upsilon_{i} M_{1 i}+X_{i} M_{2 i} & 0 & 0 & 0 & N_{1 i}^{T} & N_{3 i}^{T} & I & I & 0
\end{array}\right],
$$$$
U_{2}=\left[\begin{array}{lllllllll}
0 & 0 & 0 & 0 & N_{2 i}^{T} & 0 & 0 & 0 & 0
\end{array}\right] \text {, }
$$$$
U_{3}=\left[\begin{array}{ccccccccc}
0 & 0 & 0 & 0 & 0 & 0 & 0 & 0 & \Omega_{i} \\
0 & 0 & 0 & 0 & 0 & 0 & 0 & 0 & I
\end{array}\right] \text {, }
$$$$
U_{4}=\left[\begin{array}{ccccccccc}
M_{1 i} & 0 & 0 & 0 & 0 & 0 & 0 & 0 & 0 \\
\Upsilon_{i} M_{1 i}+X_{i} M_{2 i} & 0 & S_{i}^{T} \Upsilon_{i}+G_{i}^{T} X_{i}^{T} & 0 & 0 & 0 & 0 & 0 & 0
\end{array}\right],
$$$$
U_{5}=\left[\begin{array}{ccccccccc}
0 & M_{1 i} & 0 & 0 & 0 & 0 & 0 & 0 & 0 \\
0 & \Upsilon_{i} M_{1 i}+X_{i} M_{2 i} & 0 & 0 & 0 & 0 & 0 & 0 & 0
\end{array}\right] \text {, }
$$$$
V=\operatorname{diag}\left\{-\varepsilon I,-\varepsilon I,-I,-I,-\varepsilon^{-1} I,-\varepsilon^{-1} I,-Q_{i}^{-1},-\tau^{-1} Z_{i}^{-1}\right. \text {, }
$$$$
\left.-R_{i}^{-1}\right\}
$$

Moreover, if the above condition is feasible, then a DOF passive controller realization is given by

$$
\begin{gathered}
A_{k i}=P_{i 12}^{-1}\left(T_{i}-\Upsilon_{i} A_{i} \Omega_{i}-X_{i} C_{2 i} \Omega_{i}-\Upsilon_{i} B_{i} Y_{i}\right) W_{i 12}^{-T} \\
B_{k i}=P_{i 12}^{-1} X_{i}, \quad C_{k i}=Y_{i} W_{i 12}^{-T},
\end{gathered}
$$

where $P_{i 12}$ and $W_{i 12}$ are any nonsingular matrices and satisfy

$$
\Omega_{i} \Upsilon_{i}+W_{i 12} P_{i 12}^{T}=I .
$$

Proof. According to Theorem 12, let the matrices $P_{i}$ and $P_{i}^{-1}$ be partitioned as

$$
P_{i}:=\left[\begin{array}{cc}
P_{i 11} & P_{i 12} \\
* & P_{i 22}
\end{array}\right], \quad P_{i}^{-1}:=\left[\begin{array}{cc}
W_{i 11} & W_{i 12} \\
* & W_{i 22}
\end{array}\right],
$$


where $P_{i 11} \in R^{n} \times R^{n}, W_{i 11} \in R^{n} \times R^{n}$, and $P_{i 12}, W_{i 12}$ are nonsingular matrices. Then, define the following matrices:

$$
J_{i}:=\left[\begin{array}{ll}
W_{i 11} & I \\
W_{i 12}^{T} & 0
\end{array}\right], \quad \widetilde{J}_{i}:=\left[\begin{array}{cc}
I & P_{i 11} \\
0 & P_{i 12}^{T}
\end{array}\right] .
$$

From $P_{i}^{-1} P_{i}=I$, we have

$$
\begin{aligned}
& W_{i 11} P_{i 11}+W_{i 12} P_{i 12}^{T}=I, \\
& W_{i 12}^{T} P_{i 11}+W_{i 22} P_{i 12}^{T}=0,
\end{aligned}
$$

and $P_{i} J_{i}=\widetilde{J}_{i}, P_{i}^{-1} \widetilde{J}_{i}=J_{i}$.

By using Lemmas 7 and 8 and Schur complement lemma, we can get that (31) is equivalent to the following inequality:

$$
\left[\begin{array}{ccccccccc}
\Sigma_{11} & \Sigma_{12} & 0 & P_{i} B_{c v i}-C_{c i}^{T} & S_{c i}^{T} P_{i} & A_{c i}^{T} P_{i} & 0 & P_{i} \widetilde{M}_{1 i} & 0 \\
* & \Sigma_{22} & 0 & 0 & 0 & A_{c d i}^{T} P_{i} & 0 & 0 & 0 \\
* & * & \Sigma_{33} & 0 & 0 & 0 & L_{c i} & 0 & 0 \\
* & * & * & -D_{c v i}^{T}-D_{c v i} & 0 & B_{c v i}^{T} P_{i} & 0 & 0 & 0 \\
* & * & * & * & -P_{i} & 0 & 0 & P_{i} \widetilde{M}_{1 i} & 0 \\
* & * & * & * & * & -P_{i} & 0 & 0 & 0 \\
* & * & * & * & * & * & -P_{i} & 0 & P_{i} \widetilde{M}_{1 i} \\
* & * & * & * & * & * & * & -\varepsilon I & 0 \\
* & * & * & * & * & * & * & * & -\varepsilon I
\end{array}\right]
$$

where

$$
\begin{gathered}
\Sigma_{11}=H^{T} Q_{i} H+\tau H^{T} Z_{i} H+A_{c i}^{T} P_{i}+P_{i} A_{c i}+\varepsilon H^{T} \\
\times\left(N_{1 i}^{T} N_{1 i}+N_{3 i}^{T} N_{3 i}\right) H, \\
\Sigma_{12}=P_{i} A_{c d i}+\varepsilon H^{T} N_{1 i}^{T} N_{2 i}, \\
\Sigma_{22}=-(1-\mu) Q_{i}+\varepsilon N_{2 i}^{T} N_{2 i}, \\
\Sigma_{33}=\tau H^{T} R_{i} H-L_{i}^{T}-L_{i} .
\end{gathered}
$$

Pre- and post-multiplying (46) by matrices $\operatorname{diag}\left\{J_{i}^{T}, I, J_{i}^{T}\right.$, $\left.I, J_{i}^{T}, J_{i}^{T}, J_{i}^{T}, I, I, I, I\right\} \quad$ and $\operatorname{diag}\left\{J_{i}, I, J_{i}, I, J_{i}, J_{i}, J_{i}, I, I, I, I\right\}$, respectively, and denoting $L_{i}:=\beta P_{i}, \Omega_{i}:=W_{i 11}, \Upsilon_{i}:=P_{i 11}$, $X_{i}:=P_{i 12} B_{k i}, Y_{i}:=C_{k i} W_{i 12}^{T}$, and

$$
\begin{aligned}
T_{i}:= & \left(P_{i 11} A_{i}+P_{i 12} B_{k i} C_{2 i}\right) W_{i 11}+P_{i 11} B_{i} C_{k i} W_{i 12}^{T} \\
& +P_{i 12} A_{k i} W_{i 12}^{T},
\end{aligned}
$$

we have

$$
\left[\begin{array}{ccccccccc}
\widetilde{\Psi}_{11} & \widetilde{\Psi}_{12} & 0 & \Psi_{14} & \widetilde{\Psi}_{15} & \Psi_{16} & 0 & \Psi_{18} & 0 \\
* & \widetilde{\Psi}_{22} & 0 & 0 & 0 & \Psi_{26} & 0 & 0 & 0 \\
* & * & \Psi_{33} & 0 & 0 & 0 & -\beta \psi & 0 & 0 \\
* & * & * & \Psi_{44} & 0 & \Psi_{46} & 0 & 0 & 0 \\
* & * & * & * & \psi & 0 & 0 & \Psi_{58} & 0 \\
* & * & * & * & * & \psi & 0 & 0 & 0 \\
* & * & * & * & * & * & \psi & 0 & \Psi_{79} \\
* & * & * & * & * & * & * & -\varepsilon I & 0 \\
* & * & * & * & * & * & * & * & -\varepsilon I
\end{array}\right]<0,
$$

where

$$
\begin{aligned}
& \widetilde{\Psi}_{11}=\Psi_{11}+\varepsilon\left[\begin{array}{c}
\Omega_{i} \\
I
\end{array}\right]\left(Q_{i}+\tau Z_{i}+N_{1 i}^{T} N_{1 i}+N_{3 i}^{T} N_{3 i}\right)\left[\Omega_{i} I\right] \\
& \widetilde{\Psi}_{12}=\Psi_{12}+\varepsilon\left[\begin{array}{c}
\Omega_{i} \\
I
\end{array}\right] N_{1 i}^{T} N_{2 i}, \\
& \widetilde{\Psi}_{22}=\Psi_{22}+\varepsilon N_{2 i}^{T} N_{2 i}, \\
& \widetilde{\Psi}_{15}=\left[\begin{array}{cc}
\Omega_{i} S_{i}^{T} & \Omega_{i} S_{i}^{T} \Upsilon_{i}+\Omega_{i} G_{i}^{T} X_{i}^{T} \\
S_{i}^{T} & S_{i}^{T} \Upsilon_{i}+G_{i}^{T} X_{i}^{T}
\end{array}\right], \\
& \Psi_{33}=\beta \psi+\varepsilon\left[\begin{array}{c}
\Omega_{i} \\
I
\end{array}\right] R_{i}\left[\begin{array}{ll}
\Omega_{i} & I
\end{array}\right], \\
& \Psi_{18}=\Psi_{58}=\Psi_{79}=\left[\begin{array}{c}
M_{1 i} \\
\Upsilon_{i} M_{1 i}+X_{i} M_{2 i}
\end{array}\right] .
\end{aligned}
$$

Applying Schur complement lemma, one obtains

$$
\left.\begin{array}{ccccccccc}
\widetilde{\Psi}_{11} & \widetilde{\Psi}_{12} & 0 & \Psi_{14} & \widetilde{\Psi}_{15} & \Psi_{16} & 0 & \Psi_{18} & 0 \\
* & \widetilde{\Psi}_{22} & 0 & 0 & 0 & \Psi_{26} & 0 & 0 & 0 \\
* & * & \Psi_{33} & 0 & 0 & 0 & -\beta \psi & 0 & 0 \\
* & * & * & \Psi_{44} & 0 & \Psi_{46} & 0 & 0 & 0 \\
* & * & * & * & \psi & 0 & 0 & \Psi_{58} & 0 \\
* & * & * & * & * & \psi & 0 & 0 & 0 \\
* & * & * & * & * & * & \psi & 0 & \Psi_{79} \\
* & * & * & * & * & * & * & -\varepsilon I & 0 \\
* & * & * & * & * & * & * & * & -\varepsilon I
\end{array}\right]
$$

where

$$
\begin{aligned}
v & =\left[\begin{array}{llllllllllllll}
\Omega_{i} & 0 & 0 & 0 & 0 & 0 & 0 & 0 & 0 & 0 & 0 & 0 & 0 & 0
\end{array}\right], \\
\vartheta & =\left[\begin{array}{llllllllllllll}
0 & 0 & 0 & 0 & 0 & 0 & 0 & S_{i}^{T} \Upsilon_{i}+G_{i}^{T} X_{i}^{T} & 0 & 0 & 0 & 0 & 0 & 0
\end{array}\right] .
\end{aligned}
$$

Then by Lemma 7 and Schur complement lemma, we can obtain (39) and the parameters of the designed passive controller.

Remark 14. It should be noted that the dissipativity-based sliding mode control of switched stochastic delay-free systems was studied in [38]. However, the focus of our work is on DOF passive controller design for switched stochastic delay systems, and this is also the major contribution of the paper.

Remark 15. Theorem 13 proposes a design method of the DOF passive controller for system (1). For the prescribed positive definite symmetric matrices $Q_{i}, R_{i}$, and $Z_{i}$, we can solve the inequality (39) easily by LMI Toolbox in MATLAB. However, when these matrices are unknown, the inequality is no longer a LMI. In this case, we can use an iterative algorithm involving a nonlinear optimization problem with LMI constraint in [20] to solve it. 
The procedure of the passive controller design can be concluded as follows:

(1) by solving LMI (39), we can get the feasible solution of the positive definite symmetric matrices $\Omega_{i}, \Upsilon_{i}, Q_{i}$, $R_{i}$, and $Z_{i}$ and any matrices $X_{i}, Y_{i}$, and $T_{i}$.

(2) Applying singular value decomposition to (42), we can obtain the nonsingular matrices $P_{i 12}, W_{i 12}$.

(3) According to (41), we can calculate the controller parameters $A_{k i}, B_{k i}$, and $C_{k i}$.

\section{Numerical Example}

In this section, we present a numerical example to show the effectiveness of the proposed design method in the previous section. Consider system (1) with parameters as follows:

Subsystem 1 .

$$
\begin{gathered}
A_{1}=\left[\begin{array}{ll}
0.2 & 0.3 \\
0.1 & 0.5
\end{array}\right], \quad A_{d 1}=\left[\begin{array}{cc}
0.1 & 0 \\
0 & 0.4
\end{array}\right], \\
B_{1}=\left[\begin{array}{l}
0.2 \\
0.6
\end{array}\right], \quad B_{v 1}=\left[\begin{array}{c}
0.3 \\
-0.1
\end{array}\right], \\
S_{1}=\left[\begin{array}{cc}
-0.4 & -1 \\
-1 & 0.2
\end{array}\right], \quad C_{21}=\left[\begin{array}{cc}
0.1 & -0.4 \\
-0.2 & 0.6
\end{array}\right], \\
C_{2 d 1}=\left[\begin{array}{cc}
0.3 & -0.2 \\
-0.4 & 0.5
\end{array}\right], \quad D_{2 v 1}=\left[\begin{array}{c}
0.1 \\
-0.5
\end{array}\right], \\
G_{d 1}=\left[\begin{array}{ll}
-0.2 & 0.5 \\
-0.1 & 0.3
\end{array}\right], \quad C_{11}=[0.10 .8], \\
D_{11}=0.5, \quad D_{1 v 1}=0.4, \\
M_{11}=M_{21}=\operatorname{diag}\{0.1,0.1\}, \\
N_{11}=N_{21}=N_{31}=\operatorname{diag}\{0.2,0.2\}, \\
F_{1}=\operatorname{diag}\{\sin (0.01 t), \sin (0.01 t)\} .
\end{gathered}
$$

Subsystem 2.

$$
\begin{gathered}
A_{2}=\left[\begin{array}{cc}
-0.1 & 0.5 \\
-0.8 & 0.6
\end{array}\right], \quad A_{d 2}=\left[\begin{array}{cc}
0.3 & 0 \\
0 & 0.9
\end{array}\right], \\
B_{2}=\left[\begin{array}{c}
0.5 \\
-0.1
\end{array}\right], \quad B_{v 2}=\left[\begin{array}{c}
-0.1 \\
0.2
\end{array}\right], \\
S_{2}=\left[\begin{array}{cc}
-0.2 & 0 \\
0 & 0.1
\end{array}\right], \quad C_{22}=\left[\begin{array}{cc}
0.1 & 0 \\
0.1 & 0.5
\end{array}\right], \\
C_{2 d 2}=\left[\begin{array}{cc}
-0.3 & 0 \\
0 & -0.4
\end{array}\right], \quad D_{2 v 2}=\left[\begin{array}{c}
-0.1 \\
0.6
\end{array}\right], \\
G_{2}=\left[\begin{array}{cc}
0.6 & 0.2 \\
-0.3 & 0.5
\end{array}\right], \quad C_{12}=\left[\begin{array}{ll}
0.2 & 0.3
\end{array}\right], \\
D_{12}=0.8, \quad D_{1 v 2}=0.6, \\
M_{12}=M_{22}=\operatorname{diag}\{0.2,0.2\}, \\
N_{12}=N_{22}=N_{32}=\operatorname{diag}\{0.1,0.1\}, \quad \tau=0.5 .
\end{gathered}
$$

Choose $\varepsilon_{1}=\varepsilon_{2}=1$ and matrices $Q_{1}, Q_{2}, R_{1}, R_{2}, Z_{1}$, and $Z_{2}$ as follows:

$$
\begin{array}{ll}
Q_{1}=\operatorname{diag}\{0.5,0.5\}, & Q_{2}=\operatorname{diag}\{0.2,0.2\}, \\
R_{1}=\operatorname{diag}\{0.1,0.1\}, & R_{2}=\operatorname{diag}\{0.2,0.2\}, \\
Z_{1}=\operatorname{diag}\{0.1,0.1\}, & Z_{2}=\operatorname{diag}\{0.4,0.4\} .
\end{array}
$$

In this case, solving (39) in Theorem 13, we can obtain

$$
\begin{array}{ll}
\Omega_{1}=\left[\begin{array}{ll}
0.2537 & 0.0407 \\
0.0407 & 0.7178
\end{array}\right], & \Omega_{2}=\left[\begin{array}{ll}
0.5373 & 0.2054 \\
0.2054 & 0.2056
\end{array}\right], \\
\Upsilon_{1}=\left[\begin{array}{cc}
0.2688 & -0.2717 \\
-0.2717 & 0.4615
\end{array}\right], & \Upsilon_{2}=\left[\begin{array}{cc}
1.6648 & -0.2134 \\
-0.2134 & 0.3725
\end{array}\right], \\
X_{1}=\left[\begin{array}{cc}
0.3467 & 0.6160 \\
-0.1359 & -1.8058
\end{array}\right], & X_{2}=\left[\begin{array}{ll}
-0.2034 & -0.2568 \\
-0.0305 & -0.6650
\end{array}\right], \\
Y_{1}=\left[\begin{array}{ll}
-0.7717 & -3.4812
\end{array}\right], & Y_{2}=\left[\begin{array}{ll}
-1.9639 & 0.2288
\end{array}\right], \\
T_{1}=\left[\begin{array}{cc}
-0.5048 & 0.2014 \\
-0.3514 & -1.0718
\end{array}\right], & T_{2}=\left[\begin{array}{ll}
-0.4001 & 0.6342 \\
-0.3454 & -1.4366
\end{array}\right] .
\end{array}
$$

Then from (42), matrices $P_{112}, W_{112}, P_{212}$, and $W_{212}$ can be obtained:

$\begin{array}{ll}P_{112}=\left[\begin{array}{cc}-0.9484 & -0.3170 \\ -0.3170 & 0.9484\end{array}\right], & W_{112}=\left[\begin{array}{cc}-0.9101 & -0.2513 \\ -0.3901 & 0.5864\end{array}\right], \\ P_{212}=\left[\begin{array}{cc}-0.2956 & 0.9553 \\ 0.9553 & 0.2956\end{array}\right], & W_{212}=\left[\begin{array}{cc}-0.0077 & 0.1539 \\ 1.0121 & 0.0012\end{array}\right] .\end{array}$

From (41), we can get the DOF passive controller parameters as follows:

$$
\begin{array}{ll}
A_{k 1}=\left[\begin{array}{cc}
-0.0413 & -2.3349 \\
2.1534 & -9.1815
\end{array}\right], & B_{k 1}=\left[\begin{array}{ll}
-0.2857 & -0.0119 \\
-0.2388 & -1.9080
\end{array}\right], \\
C_{k 1}=\left[\begin{array}{ll}
2.1013 & -4.5387
\end{array}\right], & A_{k 2}=\left[\begin{array}{cc}
-1.3064 & -4.8026 \\
-0.0733 & 6.5615
\end{array}\right], \\
B_{k 2}=\left[\begin{array}{cc}
0.0310 & -0.5594 \\
-0.2034 & -0.4419
\end{array}\right], & C_{k 2}=\left[\begin{array}{ll}
0.2407 & -12.7494
\end{array}\right] .
\end{array}
$$

Let the disturbance input $v(t)=0.02 e^{-0.1 t} \sin (t)$; simulation results are shown in Figures $1-3$, where the initial states $x(0)=\left[\begin{array}{ll}1 & -2\end{array}\right]^{T}$ and $\widehat{x}(0)=\left[\begin{array}{ll}0 & 0\end{array}\right]^{T}$. The switching signal is depicted in Figure 1. Under the obtained passivity-based controller, trajectories of states $x_{1}$ and $x_{2}$ are shown in Figures 2 and 3 , respectively. From simulation results, we can see that the designed passivity-based controller can guarantee that the corresponding closed-loop system is stable; this shows the effectiveness of the proposed method.

\section{Conclusions}

This paper has investigated the problem of DOF passive control for a class of switched stochastic systems with timevarying delay. By utilizing MSFs method, a delay-dependent 


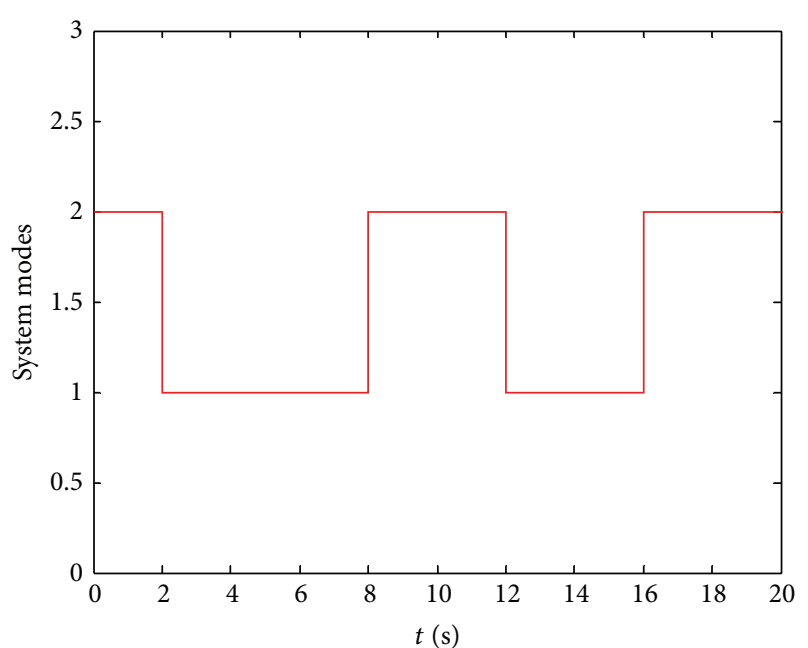

Figure 1: Switching signal.

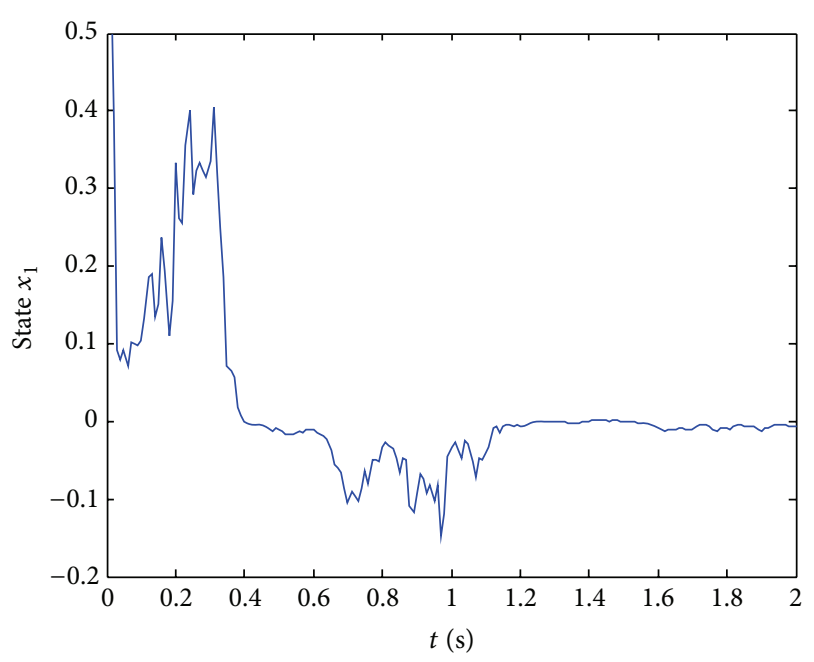

FIgURE 2: The trajectory of state $x_{1}$.

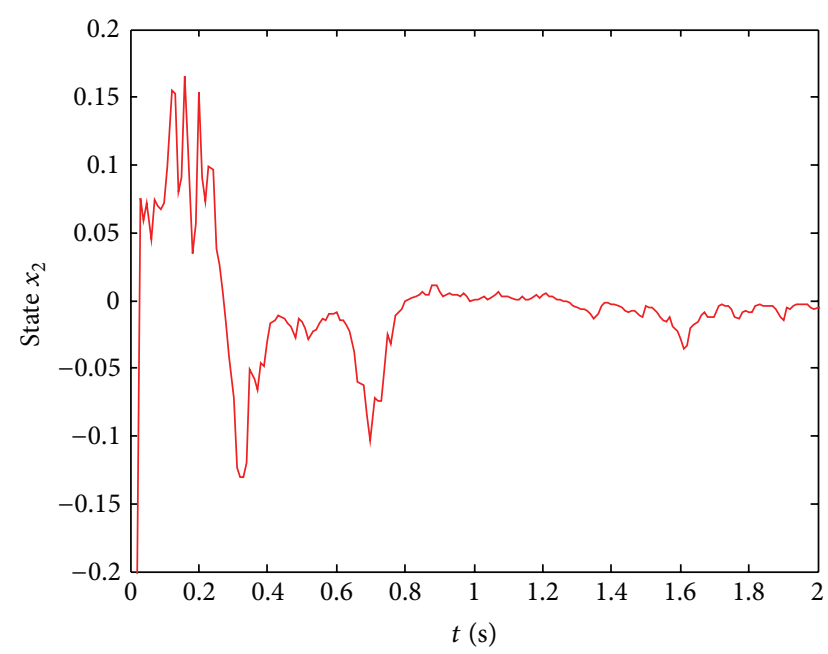

FIgURE 3: The trajectory of state $x_{2}$. sufficient condition for the existence of stochastic passivity was derived in terms of LMIs. In addition, a DOF passive controller design method was proposed. A numerical example was presented to illustrate the effectiveness of the proposed method.

\section{Acknowledgments}

This work was supported by the National Natural Science Foundation of China under Grants nos. 60974027 and 61273120 .

\section{References}

[1] S. Engell, S. Kowalewski, C. Schulz, and O. Stursberg, "Continuous-discrete interactions in chemical processing plants," Proceedings of the IEEE, vol. 88, no. 7, pp. 1050-1068, 2000.

[2] Y. Q. Shi, "The theory of hybrid control systems and its application perspective in electric power systems," in Proceedings of the International Conferences on Info-tech and Info-net (ICII'01), vol. 4, pp. 85-94, Beijing, China, 2001.

[3] K. S. Narendra, O. A. Driollet, M. Feiler, and K. George, "Adaptive control using multiple models, switching and tuning," International Journal of Adaptive Control and Signal Processing, vol. 17, no. 2, pp. 87-102, 2003.

[4] E. Skafidas, R. J. Evans, A. V. Savkin, and I. R. Petersen, "Stability results for switched controller systems," Automatica, vol. 35, no. 4, pp. 553-564, 1999.

[5] X.-M. Sun, J. Zhao, and D. J. Hill, "Stability and $L_{2}$-gain analysis for switched delay systems: a delay-dependent method," Automatica, vol. 42, no. 10, pp. 1769-1774, 2006.

[6] Z. Xiang, Y.-N. Sun, and Q. Chen, "Robust reliable stabilization of uncertain switched neutral systems with delayed switching," Applied Mathematics and Computation, vol. 217, no. 23, pp. 9835-9844, 2011.

[7] Z. R. Xiang and R. H. Wang, "Robust stabilization of switched non-linear systems with time-varying delays under asynchronous switching," Journal of Systems and Control Engineering, vol. 223, no. 8, pp. 1111-1128, 2009.

[8] L. Wu, D. W. C. Ho, and C. W. Li, "Sliding mode control of switched hybrid systems with stochastic perturbation," Systems \& Control Letters, vol. 60, no. 8, pp. 531-539, 2011.

[9] L. Wu, X. Su, and P. Shi, "Sliding mode control with bounded $L_{2}$ gain performance of Markovian jump singular time-delay systems," Automatica, vol. 48, no. 8, pp. 1929-1933, 2012.

[10] Z. He, J. Wu, S. Sun, and C. Gao, "State estimation and sliding mode control of uncertain switched hybrid systems, International," Journal of Innovative Computing, Information and Control, vol. 8, no. 10 B, pp. 7143-7156, 2012.

[11] H. R. Shaker and R. Wisniewski, "Model reduction of switched systems based on switching generalized gramians, International," Journal of Innovative Computing, Information and Control, vol. 8, no. 7 B, pp. 5025-5044, 2012.

[12] L. Wu and W. X. Zheng, "Weighted $H_{\infty}$ model reduction for linear switched systems with time-varying delay," Automatica, vol. 45, no. 1, pp. 186-193, 2009.

[13] Z. Xiang, C. Liang, and M. S. Mahmoud, "Robust $L_{2}-L_{\infty}$ filtering for switched time-delay systems with missing measurements," Circuits, Systems, and Signal Processing, vol. 31, no. 5, pp. 1677-1697, 2012. 
[14] L. Wu and Z. Wang, "Guaranteed cost control of switched systems with neutral delay via dynamic output feedback," International Journal of Systems Science, vol. 40, no. 7, pp. 717728, 2009.

[15] J. C. Geromel, P. Colaneri, and P. Bolzern, "Dynamic output feedback control of switched linear systems," IEEE Transactions on Automatic Control, vol. 53, no. 3, pp. 720-733, 2008.

[16] L. Wu, D. W. C. Ho, and C. W. Li, "H ${ }_{\infty}$ dynamic output feedback control of switched stochastic systems," in Proceedings of the 48th IEEE Conference on Decision and Control and 28th Chinese Control Conference (CDC/CCC '09), pp. 500-505, Shanghai, China, December 2009.

[17] L. Hou, G. Zhong, and Y. Wu, "Finite-time control for switched delay systems via dynamic output feedback," International Journal of Innovative Computing Information and Control, vol. 8, no. 7 A, pp. 4901-4913, 2012.

[18] H. Lin and P. J. Antsaklis, "Stability and stabilizability of switched linear systems: a survey of recent results," IEEE Transactions on Automatic Control, vol. 54, no. 2, pp. 308-322, 2009.

[19] W.-H. Chen, Z.-H. Guan, and X. Lu, "Delay-dependent exponential stability of uncertain stochastic systems with multiple delays: an LMI approach," Systems \& Control Letters, vol. 54, no. 6, pp. 547-555, 2005.

[20] S. Xu, Y. Chu, J. Lu, and Y. Zou, "Exponential dynamic output feedback controller design for stochastic neutral systems with distributed delays," IEEE Transactions on Systems, Man, and Cybernetics A, vol. 36, no. 3, pp. 540-548, 2006.

[21] L. Wu, D. W. C. Ho, and C. W. Li, "Stabilisation and performance synthesis for switched stochastic systems," IET Control Theory \& Applications, vol. 4, no. 10, pp. 1877-1888, 2010.

[22] V. Filipovic, "Exponential stability of stochastic switched systems," Transactions of the Institute of Measurement and Control, vol. 31, no. 2, pp. 205-212, 2009.

[23] W. Feng, J. Tian, and P. Zhao, "Stability analysis of switched stochastic systems," Automatica, vol. 47, no. 1, pp. 148-157, 2011.

[24] R. Yang, Z. Zhang, and P. Shi, "Exponential stability on stochastic neural networks with discrete interval and distributed delays," IEEE Transactions on Neural Networks, vol. 21, no. 1, pp. 169-175, 2010.

[25] R. Yang, H. Gao, and P. Shi, "Novel robust stability criteria for stochastic Hopfield neural networks with time delays," IEEE Transactions on Systems, Man, and Cybernetics B, vol. 39, no. 2, pp. 467-474, 2009.

[26] X. D. Zhao, L. Wang, M. X. Ling, and Q. S. Zeng, "Less conservative delay-dependent exponential stability for stochastic Markovian jump systems with time-varying delays," Journal of Harbin Institute of Technology, vol. 42, no. 3, pp. 378-383, 2010.

[27] X. Su, P. Shi, L. Wu, and S. K. Nguang, "Induced $l_{2}$ filtering of fuzzy stochastic systems with time-varying delays," IEEE Transactions on Systems, Man and Cybernetics B, vol. PP, no. 99, pp. 1-14, 2012.

[28] M. S. Branicky, "Multiple Lyapunov functions and other analysis tools for switched and hybrid systems," IEEE Transactions on Automatic Control, vol. 43, no. 4, pp. 475-482, 1998.

[29] G. Zhai, B. Hu, K. Yasuda, and A. N. Michel, "Stability analysis of switched systems with stable and unstable subsystems: an average dwell time approach," in Proceedings of the American Control Conference, pp. 200-204, Chicago, Ill, USA, June 2000.

[30] D. Liberzon and A. S. Morse, "Basic problems in stability and design of switched systems," IEEE Control Systems Magazine, vol. 19, no. 5, pp. 59-70, 1999.
[31] J. C. Willems, "Dissipative dynamical systems-part I: general theory," Archive for Rational Mechanics and Analysis, vol. 45, no. 5, pp. 321-351, 1972.

[32] D. Hill and P. Moylan, "The stability of nonlinear dissipative systems," IEEE Transactions on Automatic Control, vol. AC-21, no. 5, pp. 708-711, 1976.

[33] M. Žefran, F. Bullo, and M. Stein, "A notion of passivity for hybrid systems," in Proceedings of the 40th IEEE Conference on Decision and Control (CDC '01), pp. 768-773, Orlando, Fla, USA, December 2001.

[34] J. Zhao and D. J. Hill, "Dissipativity theory for switched systems," IEEE Transactions on Automatic Control, vol. 53, no. 4, pp. 941-953, 2008.

[35] W. Chen and M. Saif, "Passivity and passivity based controller design of a class of switched control systems," in Proceedings of the 16th Triennial World Congress of International Federation of Automatic Control (IFAC '05), p. 776, Prague, Czech, July 2005.

[36] Y. Y. Liu, J. Zhao, and G. M. Dimirovski, "Passivity, feedback equivalence and stability of switched nonlinear systems using multiple storage functions," in Proceedings of the 30th Chinese Control Conference (CCC '11), pp. 1805-1809, Yantai, China, 2011.

[37] Y. Y. Liu, G. S. Stojanovski, M. J. Stankovski, G. M. Dimirovski, and J. Zhao, "Feedback passivation of switched nonlinear systems using storage-like functions," International Journal of Control, Automation, and Systems, vol. 9, no. 5, pp. 980-986, 2011.

[38] L. Wu, W. X. Zheng, and H. Gao, "Dissipativity-based sliding mode control of switched stochastic systems," IEEE Transactions on Automatic Control, vol. 58, no. 3, pp. 785-791, 2013.

[39] C. E. de Souza and X. Li, "Delay-dependent robust $H_{\infty}$ control of uncertain linear state-delayed systems," Automatica, vol. 35, no. 7, pp. 1313-1321, 1999. 


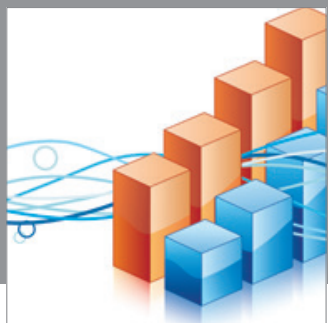

Advances in

Operations Research

mansans

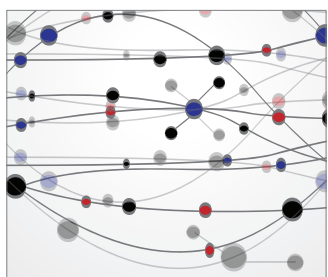

The Scientific World Journal
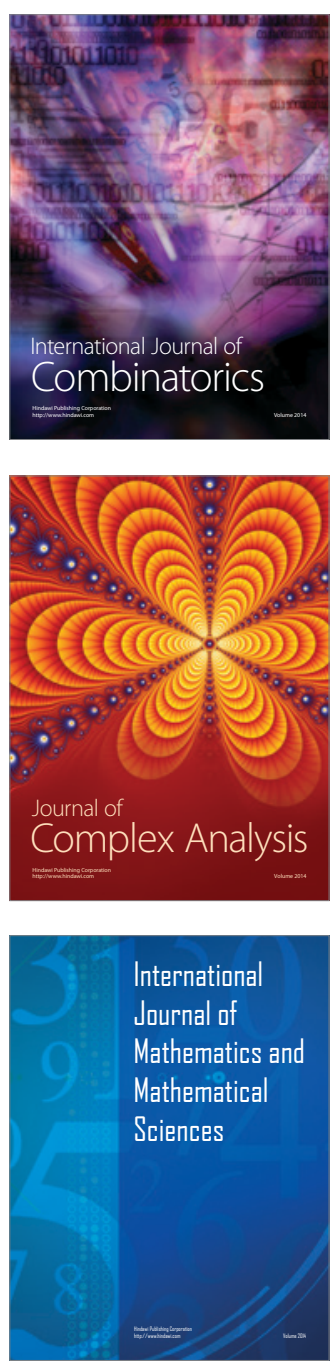
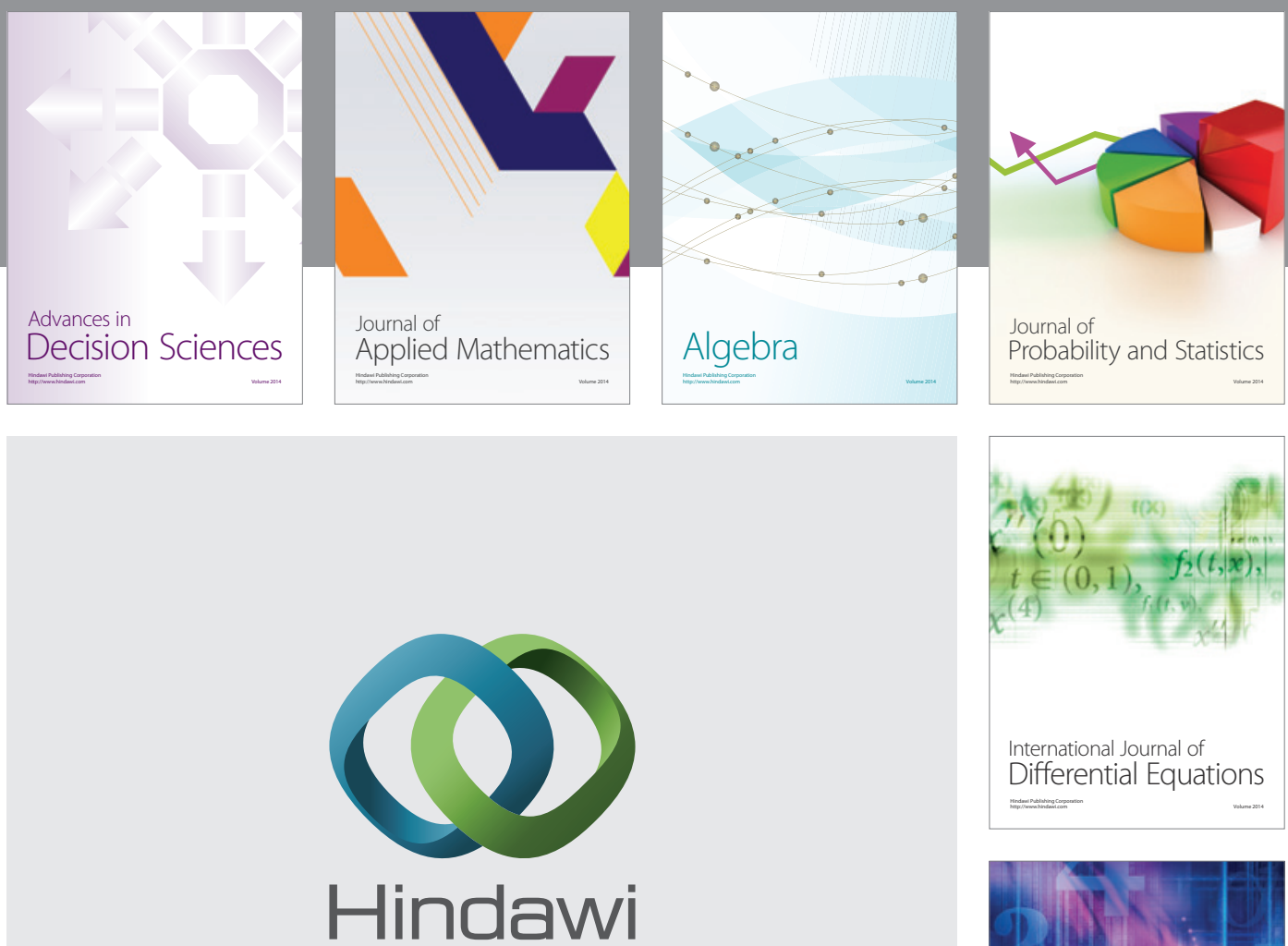

Submit your manuscripts at http://www.hindawi.com
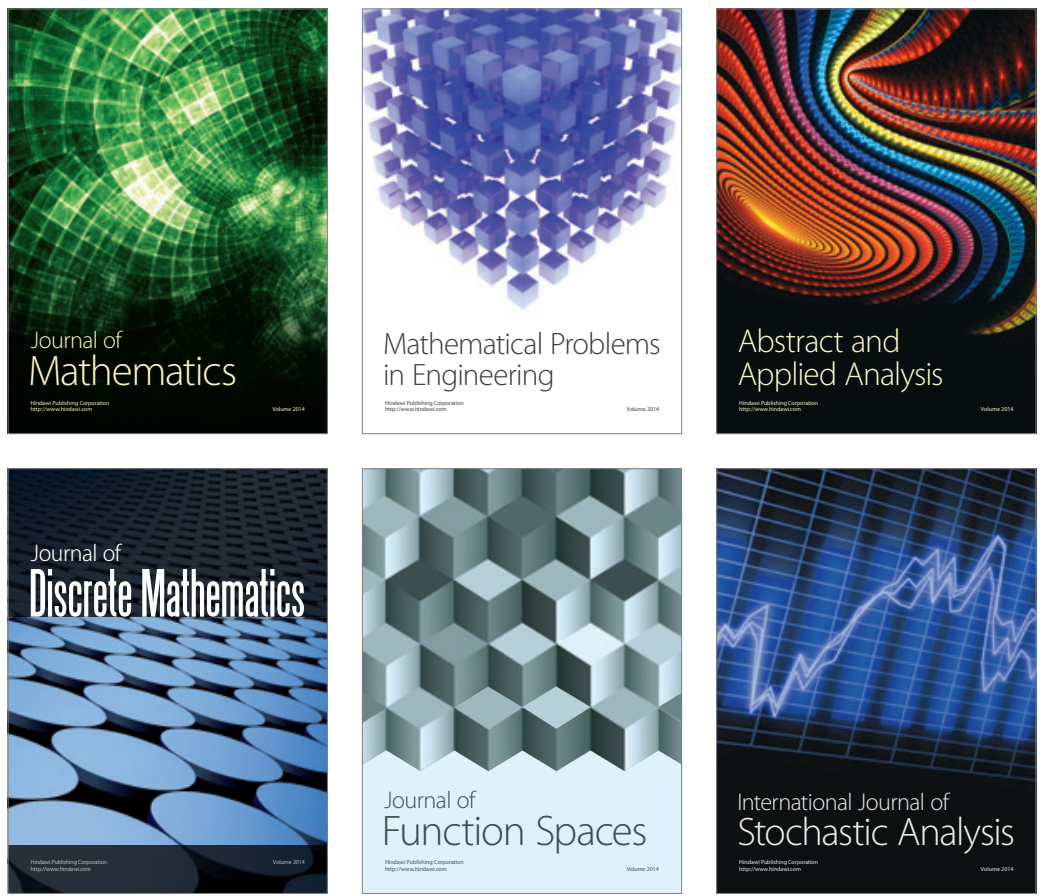

Journal of

Function Spaces

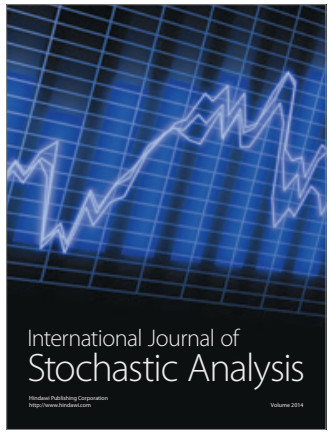

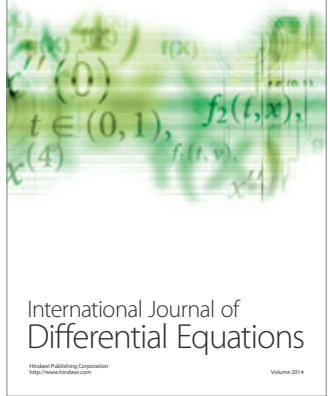
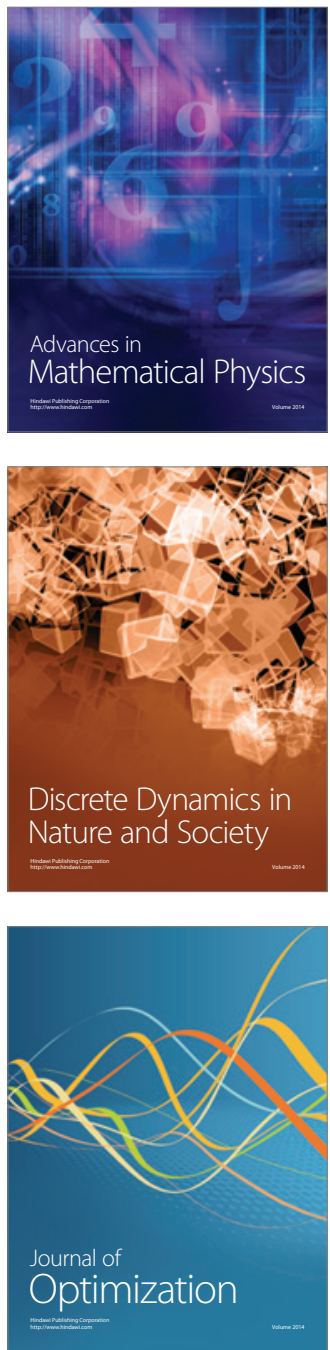